\title{
Fabrication of SiC Off-axis Aspheric Mirror by Using Robot Polishing
}

\author{
Haitao Liu*, Fengtao Yan, Wenchuan Zhao, Jieli Wu, and Min Zhou \\ Institute of Optics and Electronics, Chinese Academy of Sciences, 610209 Chengdu, China
}

\begin{abstract}
Two silicon carbide ( $\mathrm{SiC}$ ) lightweight off-axis aspheric mirrors were fabricated by using robot polishing technology. One is a primary mirror with dimension $380 \mathrm{~mm} \times 328 \mathrm{~mm}$, the other is a tertiary mirror with dimension $294 \mathrm{~mm} \times 238 \mathrm{~mm}$. The robot polishing technology is the combination of CCOS technology and a 6-axises industrial robot. The setup of robot polishing system is discussed, and the mirror aspheric grinding, surface polishing and figuring process are reported in this paper. The final surface shape error of primary mirror is $11.4 \mathrm{~nm}$ RMS, and the tertiary is $12.1 \mathrm{~nm}$ RMS. The capability of off-axis aspheric mirror fabricating on robot polishing system is verified.
\end{abstract}

\section{Introduction}

$\mathrm{SiC}$ has excellent material property such as high thermal and mechanical stability, which is suitable for optical application in aerospace and next generation lithography. Off-axis aspheric mirrors is widely used in many optical systems [1]. For $\mathrm{SiC}$ off-axis aspheric mirrors, the fabricating quality, efficiency and costs are three main problems. The Computer Controlled Optical Surfacing (CCOS) technology has long been recognized as a suitable way to grinding and polishing aspheric surface $[2$, 3].

In this paper, we introduce the setup of the robot polishing system, and present the process of $\mathrm{SiC}$ mirror aspheric grinding and polishing. And the fabrication results is shown.

\subsection{Robot polishing system}

We use a Stäubli TX200 heavy duty 6-axises industrial robot as the motion platform. The polishing tool is a spinorbital due-rotate sub-aperture lap which mounted on the robot. The polishing force is automatically applied by pneumatic cylinder in order to obtain stable force load during the polishing.

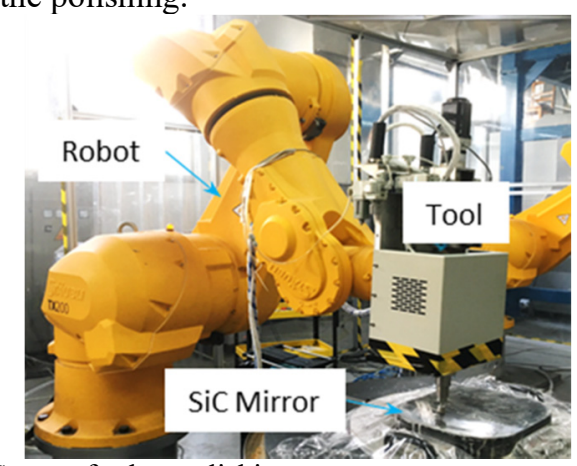

Fig. 1. Setup of robot polishing system.

In order to make the robot suitable CCOS fabrication, we developed a software in VAL3 language which runs on the robot controller in order to interpret the G-code like polishing path file to the robot moving code. below.

The specifications of robot polishing machine are list

- Maximum work range: $2 \mathrm{~m}$

- Trajectory accuracy: $\pm 0.1 \mathrm{~mm}$

- Max. tool spin speed: $300 \mathrm{rpm}$

- Max. tool orbital speed: $200 \mathrm{rpm}$

- Max. polishing force load: $30 \mathrm{~N}$

- Capability: plane, sphere, asphere and freeform surface's grinding and polishing

\subsection{Specification of the SiC mirrors}

The primary and tertiary mirror are both high order offaxis aspheric mirrors with rectangle shape. The dimension of primary mirror is $380 \mathrm{~mm} \times 328 \mathrm{~mm}$, and the tertiary mirror is $294 \mathrm{~mm} \times 238 \mathrm{~mm}$. The material is reaction burned silicon carbide (RB-SiC). The requirement of surface shape error is $\lambda / 50$ RMS for these two mirrors $(\lambda=632.8 \mathrm{~nm})$.

The initial surface is best fitting sphere, so the aspheric deviation is quite large. The initial shape error of primary mirror is $0.2 \mathrm{~mm} \mathrm{PV}$, and tertiary mirror is $0.08 \mathrm{~mm} \mathrm{PV}$. So before the polishing, the aspheric grinding is used in order the remove the large surface shape error.

\section{Aspheric grinding of $\mathrm{SiC}$ mirrors}

In grinding, the copper pad is used in order to remove the bulk errors, tool marks and sub-surface damages. The pad size is from $15 \mathrm{~mm}$ to $50 \mathrm{~mm}$ in diameter, determined by the spatial frequency of the surface shape error that need to be removed. The diamond abrasives with different particle size are used in different phase. There are 4 size abrasives, which are W40, W20, W10 and W5, has been used in grinding.

\footnotetext{
*orresponding author: 1htioe@163.com
} 
The surface shape is measured by LEITZ ultra CMM in the grinding. We developed a program to analysis the error map by using the multi dimension optimize algorithm. The surface shape error can be reduced to about 2 um PV after grinding process, see figure 2.
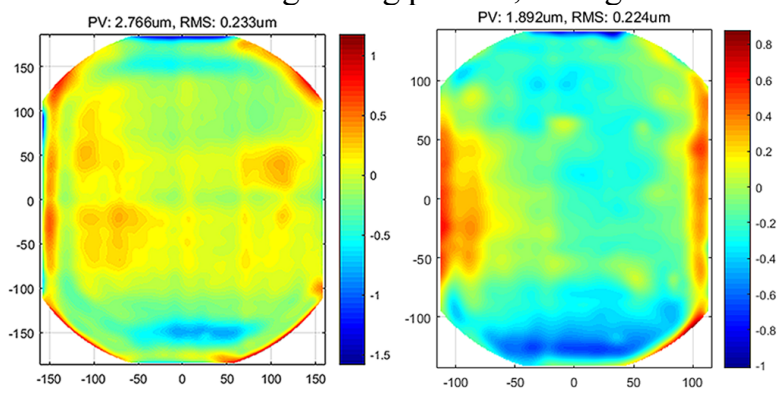

Fig. 2. Surface shape error after grinding. (Left: primary mirror, PV 2.8um. Right: tertiary mirror, PV 1.9um)

\section{Aspheric polishing of $\mathrm{SiC}$ mirrors}

Polishing process includes $\mathrm{SiC}$ substrate polishing before surface modification, Si substrate polishing after surface modification, and surface figuring. Surface modification process is an efficient way that can improving $\mathrm{SiC}$ surface compactness by depositing a thin layer of $\mathrm{Si}$ in the surface.

In $\mathrm{SiC}$ substrate polishing, the material of polishing pad is polyurethane, and the abrasive is W2 diamond polishing slurry. The main goal is to polish the surface to make it suitable for surface modification, and reduce surface shape error. By using the robot polishing, the surface shape error can be reduced to $\lambda / 10 \sim \lambda / 20$ RMS, and the surface roughness can be reduced to less than $10 \mathrm{~nm}$ Ra. Figure 3 shows the polishing of primary mirror.

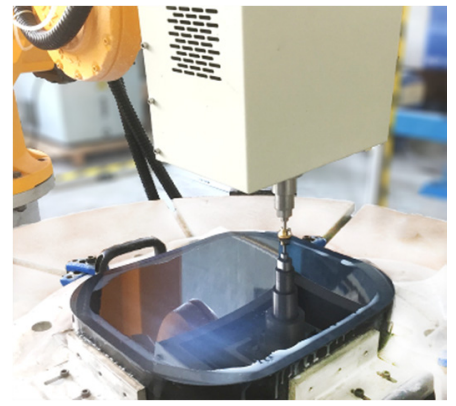

Fig. 3. Primary mirror substrate polishing by robot.

Ion beam assisted deposition method is used in surface modification. There is about $10 \mu \mathrm{m}$ thickness Si layer deposited in the $\mathrm{SiC}$ substrate surface. The surface shape error is almost maintained the same after the surface modification, but the roughness is increased due to the deposition. So the mirror surface need to be re-polishing.

The final surface figuring is completed by combine the robot polishing and magnetortheological finishing (MRF). The MRF is used to achieve high accuracy figuring and the robot polishing is used to smoothing the surface to remove middle-high spatial frequency shape error. The finished mirror surface is shown in figure 4.

During polishing, the aspheric surface shape error is measured through computer generated holograph (CGH) null-compensation interferometry. A 6 inch Zygo Verifire interferometer with $1 \mathrm{~K} \times 1 \mathrm{~K}$ pixels $C C D$ is used in the metrology.

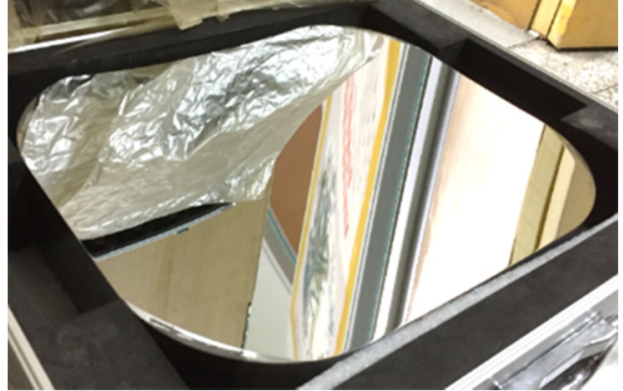

Fig. 4. The finished mirror surface. (Primary mirror)

\section{Results and discussion}

Through several iteration of MRF figuring and robot polishing, the surface shape error can be reduced to satisfied the requirement. The surface shape error for primary mirror is $11.4 \mathrm{~nm}$ RMS (see figure 5), and for tertiary mirror is $12.1 \mathrm{~nm}$ RMS (not show here).

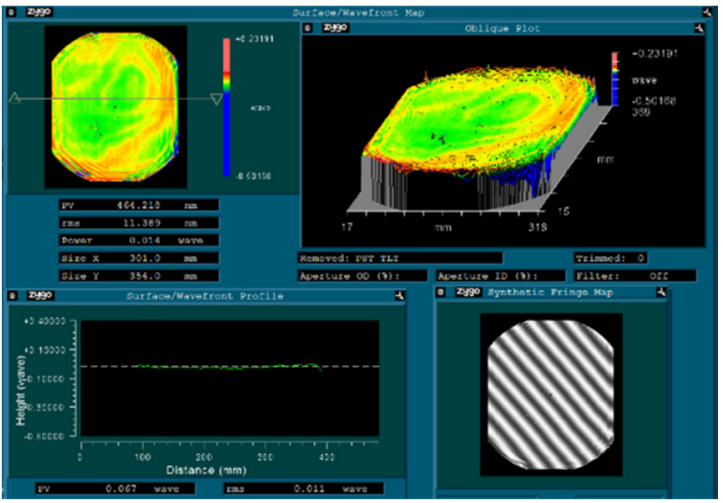

Fig. 5. Final surface shape error of primary mirror.

Surface roughness is also measured by using Bruker white light interferometer, and the roughness is about $1.5 \mathrm{~nm} \sim 3 \mathrm{~nm}$ in Ra. Figure 6 show one of the results of primary mirror.
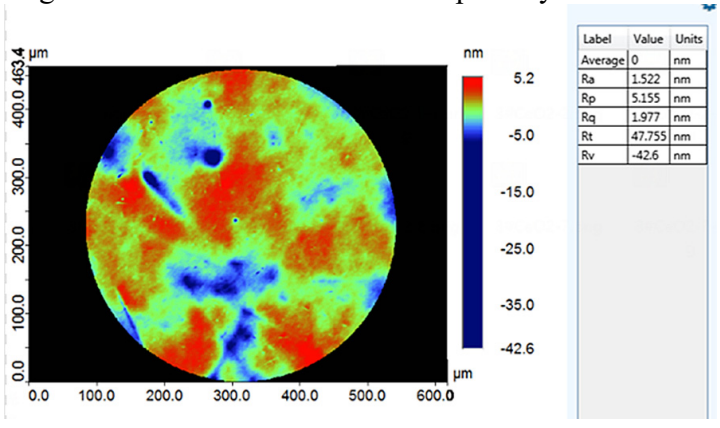

Fig. 6. Surface roughness of primary mirror.

\section{References}

1. C. Atkinson, S. Texter, Status of the JWST optical telescope element, Proc. of SPIE, Vol. 8442, 2012.

2. R. A. Jones, Fabrication of off-axis optical segments, Proc. of SPIE, vol. 1752, 1992.

3. D. D. Walker, C. Dunn, G. Yu, et. al., The role of robotics in computer controlled polishing of large and small optics, Proc. of SPIE, vol. 9575, 95750B, 2015. 